\title{
A SIRT1 agonist reduces cognitive decline in type 2 diabetic rats through antioxidative and anti-inflammatory mechanisms
}

\author{
FEI WANG ${ }^{1}$, YANCHANG SHANG ${ }^{2}$, RONG ZHANG $^{1}$, XIANGYANG GAO $^{1}$ and QIANG ZENG $^{1}$ \\ ${ }^{1}$ Health Management Institute; ${ }^{2}$ Department of Geriatric Neurology, Chinese PLA General Hospital, Beijing 100853, P.R. China
}

Received October 8, 2017; Accepted March 2, 2018

DOI: $10.3892 / \mathrm{mmr} .2018 .9699$

\begin{abstract}
Sirtuin 1 (SIRT1) is an NAD ${ }^{+}$-dependent protein deacetylase that is involved in cell differentiation, aging, apoptosis, physiological rhythms, metabolic regulation, oxidative stress and numerous other important biological processes. In the present study, the ability of a sirtuin-1 (SIRT1) agonist, SRT1720, to reduce cognitive decline in type 2 diabetes mellitus (T2DM) was investigated. Streptozotocin-induced male Sprague-Dawley rats were used to establish a T2DM model and the protective effect of SRT1720 and its underlying mechanisms were investigated. Body weight and fasting blood glucose (FBG) were recorded and cognitive function was measured with the Morris water maze. Levels of oxidative stress, inflammation, caspase-3 activity and nuclear factor $\kappa \mathrm{B}(\mathrm{NF}-\kappa \mathrm{B})$ mRNA expression were detected with a series of commercial assay kits and reverse transcription-quantitative polymerase chain reaction, respectively. Western blot analysis was performed to determine the protein expression of $\mathrm{NF}-\kappa \mathrm{B}$, endothelial nitric oxide synthase (eNOS), peroxisome proliferator-activated receptor $\gamma$ (PPAR $\gamma$ ), AMP-activated protein kinase (AMPK), heat shock $70 \mathrm{kDa}$ protein (HSP70), SIRT1, nuclear factor erythroid 2-related factor 2 (Nrf2) and heme oxygenase 1 (HO-1). The results revealed that SRT1720 significantly increased body weight, decreased FBG, improved cognitive function and reduced the levels of proteins associated with oxidative stress and inflammation damage in T2DM rats. Additionally, SRT1720 significantly decreased NF- $\kappa$ B p65 mRNA expression and increased eNOS and PPAR $\gamma$ expression. SRT1720 significantly reduced caspase-3 activity and HSP70 protein expression, and increased p-AMPK, SIRT1, Nrf2 and HO-1 protein expression. Collectively, the results indicate that SRT1720 may reduce cognitive decline in T2DM rats through antioxidative and anti-inflammatory action via NF- $\kappa \mathrm{B}$ and AMPK-dependent mechanisms.
\end{abstract}

Correspondence to: Dr Qiang Zeng, Health Management Institute, Chinese PLA General Hospital, 28 Fuxing Road, Beijing 100853, P.R. China

E-mail: zsdg2286700@126.com

Key words: sirtuin-1, cognitive decline, type 2 diabetic rats, nuclear factor- $\kappa \mathrm{B}, \mathrm{AMP}$-activated protein kinase

\section{Introduction}

Due to lifestyle changes, the prevalence of type 2 diabetes mellitus (T2DM) in China is increasing; the incidence rate of T2DM is $12 \%(1,2)$ and complications associated with T2DM, such as diabetes-associated cognitive decline (DACD), are subsequently becoming increasingly prominent. DACD has received increased attention due to its severe effects on health and the quality of life of patients, including memory deficits and neurasthenia (2).

Nuclear factor- $\kappa \mathrm{B}(\mathrm{NF}-\kappa \mathrm{B})$ combines with NF- $\mathrm{BB}$ inhibitor $\alpha(\mathrm{I} \kappa \mathrm{Ba})$ in the cytoplasm in its inactive state during T2DM/DADC. When activated, NF- $\kappa \mathrm{B}$ dissociates from

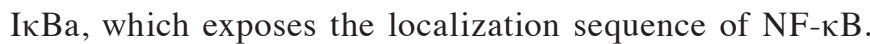
Subsequently, NF- $\kappa \mathrm{B}$ translocates to the nucleus to regulate transcription $(3,4)$. It induces the expression of proinflammatory factors, including interleukin (IL)-1, intercellular adhesion molecule 1 and tumor necrosis factor $\alpha(3,4)$.

Nuclear factor erythroid 2-related factor 2 (Nrf2) is a key anti-oxidative transcription factor in T2DM/DADC (5). Early prevention and treatment of diabetic cognitive dysfunction may delay and reduce the incidence of dementia, thus improving the quality of life of patients with diabetes (6).

Sirtuin 1 (SIRT1) is an $\mathrm{NAD}^{+}$-dependent protein deacetylase that is involved in cell differentiation, aging, apoptosis, physiological rhythms, metabolic regulation, oxidative stress and numerous other important biological processes. Furthermore, it has an important biological role in transcriptional regulation (7). Studies have demonstrated that SIRT1 is required for the maintenance of normal cognitive function and synaptic plasticity regulation. It is also reported to exhibit neuroprotective effects against Alzheimer's disease and other degenerative disease, through thermoregulation, reduction of $\mathrm{A} \beta$ protein deposition and antioxidative, anti-inflammatory and antiapoptotic mechanisms (8). The present study investigated the effect and underlying mechanisms of the SIRT1 agonist, SRT1720, on cognitive decline in a rat model of T2DM.

\section{Materials and methods}

Experimental animals. Male Sprague-Dawley rats $(\mathrm{n}=40$; weight, $210 \pm 20 \mathrm{~g} ; 8$ weeks old) were purchased from Beijing Vital River Laboratory Animal Technology Co., Ltd (Beijing, China) and housed at $23 \pm 2^{\circ} \mathrm{C}$ with $50-60 \%$ humidity on a $12 \mathrm{~h}$ light/dark cycle, free access to food and water access, with 
$\mathrm{n}=3$ rats/cage. The present study was approved by the Ethics Committee of the Chinese PLA General Hospital (Beijing, China). Rats of the control group were subjected to a single intraperitoneal injection of normal saline. Rats of T2DM model were subjected to a single intraperitoneal injection of streptozotocin saline (STZ; 65 mg/kg, Sigma-Aldrich; Merck KGaA, Darmstadt, Germany) dissolved in $100 \mathrm{mM}$ sodium citrate buffer ( $\mathrm{pH} 4.5$ ) to induce T2DM. The body weight of the T2DM rats was monitored. The fasting blood glucose (FBG) of the T2DM rats was confirmed with an Optium Xceed FBG meter (Abbott Pharmaceutical Co., Ltd., Lake Bluff, IL, USA). Rats with blood glucose levels $>300 \mathrm{mg} / \mathrm{dl}$ were considered diabetic and used in subsequent experiments. The dose of SRT1720 was selected based on a recent study (9). At 8 weeks after the development of diabetes, rats were divided into four groups: Normal control rats $(n=6)$, T2DM rats $(n=8)$, T2DM rats treated with $25 \mathrm{mg} / \mathrm{kg}$ SRT1720 (MedChemExpress, Monmouth Junction, NJ, USA) $(n=8)$ and T2DM rats treated with $50 \mathrm{mg} / \mathrm{kg}$ SRT1720 $(\mathrm{n}=8)$. Treatment group rats were administered SRT1720 via gavage for 4 weeks. Rats of the normal control group were treated with saline. Rats were anesthetized with an intraperitoneal injection of pentobarbital (35 $\mathrm{mg} / \mathrm{kg}$ ) and sacrificed by decapitation. Hippocampal samples were collected and saved at $-80^{\circ} \mathrm{C}$.

Morris water maze test. After the 4 week SRT17200 treatment period, the effect of SRT1720 on cognitive function was evaluated with the Morris water maze test. Rats were trained twice a day every day for 5 days and the test was performed in a blind fashion. Swimming was video tracked and latency, path length, swim speed and the cumulative distance from the platform were recorded. Mean swim latency was evaluated on each day. Following a probe trial, the mean time spent in the correct quadrant containing the platform and the mean number of times that mice crossed the former platform position during $60 \mathrm{sec}$ was determined for day 5 .

PC12 diabetic cell model. PC12 cells were purchased from the Type Culture Collection of the Chinese Academy of Sciences (Shanghai, China) and were cultured in Dulbecco's modified Eagle's medium (Gibco; Thermo Fisher Scientific, Inc., Waltham, MA, USA) containing 10\% fetal bovine serum (Gibco; Thermo Fisher Scientific, Inc.) and antibiotics at $37^{\circ} \mathrm{C}$ in $5 \% \mathrm{CO}_{2} . \mathrm{PC} 12$ cells were treated with $25 \mathrm{mg} / \mathrm{ml}$ glucose for $24 \mathrm{~h}$ at $37^{\circ} \mathrm{C}$. Cells were divided into four groups: Control group $(25 \mathrm{mg} / \mathrm{ml}$ glucose only), SRT1720 $(10 \mu \mathrm{M})$ group, SRT1720 $(10 \mu \mathrm{M})+\operatorname{Nrf} 2$ agonist (curcumin, $25 \mu \mathrm{M}$, MedChemExpress) group and SRT1720 $(10 \mu \mathrm{M})+\mathrm{NF}-\kappa \mathrm{B}$ inhibitor (JSH-23, $2 \mu \mathrm{M}$, MedChemExpress) group. Groups were treated for $24 \mathrm{~h}$ at $37^{\circ} \mathrm{C}$.

Measurement of oxidative stress and inflammation. Hippocampal samples or PC12 cells were homogenized and protein was extracted with radioimmunoprecipitation assay (RIPA) lysis buffer (BestBio, Shanghai, China). Levels of glutathione (GSH, S0053), GSH peroxidase (GSH-PX, S0056), superoxide dismutase (SOD, S0109), malondialdehyde (MDA, S0131), IL-1 $\beta$ (PI303) and IL-6 (PI328) were detected by their respective enzyme-linked immunosorbent assay (ELISA) kits (Beyotime Institute of Biotechnology, Nanjing, China), according to the manufacturer's protocols. Absorbency was measured at $450 \mathrm{~nm}$.

Western blot analysis. Hippocampus Samples or PC12 cell were homogenized and dissociated in RIPA lysis buffer (Beyotime Institute of Biotechnology) and protein concentration was quantified via a Bicinchoninic Acid assay (Beyotime Institute of Biotechnology). Equal amounts of protein $(50 \mu \mathrm{g})$ were separated by $12 \%$ SDS-PAGE and transferred onto polyvinylidene difluoride membranes. Subsequently, membranes were blocked in Tris buffered saline with $0.1 \%$ Tween-20 (TBST) containing 5\% milk prior for $1 \mathrm{~h}$ at $37^{\circ} \mathrm{C}$ to incubation with the following primary antibodies overnight at $4^{\circ} \mathrm{C}$ : Anti-NF-кB p65 (1:1,000; sc-71677), anti-endothelial nitric oxide synthase (eNOS, 1:1,000; sc-136977), anti-peroxisome proliferator-activated receptor $\gamma$ (PPAR $\gamma, 1: 1,000$; sc-1981), anti-AMP-activated protein kinase (AMPK, 1:1,000; cat. no. sc-74461), anti-heat shock $70 \mathrm{kDa}$ protein (HSP70, 1:1,000; sc-6242), anti-SIRT1 (1:1,000; sc-135791), anti-Nrf2 (1:1,000; sc-722), anti-heme oxygenase 1 (HO-1, 1:1,000; sc-136960) and anti- $\beta$-actin (1:5,000; sc-1615; all Santa Cruz Biotechnology, Inc., Dallas, TX, USA). Following three washes with TBST, the membrane was incubated with goat anti-rabbit IgG-horseradish peroxidase (1:5,000; sc-2004; Santa Cruz Biotechnology, Inc.) at room temperature for $2 \mathrm{~h}$ with shaking according to the manufacturer's protocols. Bands were visualized using BeyoECL Plus (Beyotime Institute of Biotechnology) and densitometry analysis was performed with Image Lab 3.0 (Bio-Rad Laboratories, Inc.).

Caspase-3 activity. Hippocampal samples or PC12 cell were homogenized and dissociated in RIPA lysis buffer. Activity was determined with a Caspase-3 Activity kit (cat. no. C1115; Beyotime Institute of Biotechnology), according to the manufacturer's protocol. The sample mixture was incubated at $37^{\circ} \mathrm{C}$ for $120 \mathrm{~min}$ and absorbance values were measured at $405 \mathrm{~nm}$.

Statistical analysis. All data are presented as the mean \pm standard deviation $(n=3)$. All statistical tests were conducted using SPSS 17.0 (SPSS, Inc., Chicago, IL, USA). Statistical significance was analyzed using one-way analysis of variance followed by Dunnett's post-hoc test. $\mathrm{P}<0.05$ was considered to indicate a statistically significant difference.

\section{Results}

SRT1720 increases body weight and reduces FBG. The body weight of the DM group was significantly decreased compared with the control group. Notably, at the end of SRT1720 treatment, the body weight of both SRT1720-treated groups was significantly increased compared with the DM group (Fig. 1A). The FBG of DM rats was significantly higher compared normal control rats. SRT1720 treatment at both concentrations significantly decreased FBG compared with the DM group (Fig. 1B). In the DM group, the number of neurocyte was reduced, compared with in the control group; treatment with SRT1720 appeared to have increased the number of neurocyte compared with the DM group (Fig. 1C). These results indicated that SRT1720 treatment may increase body weight and reduce $\mathrm{FBG}$ in T2DM rats. 

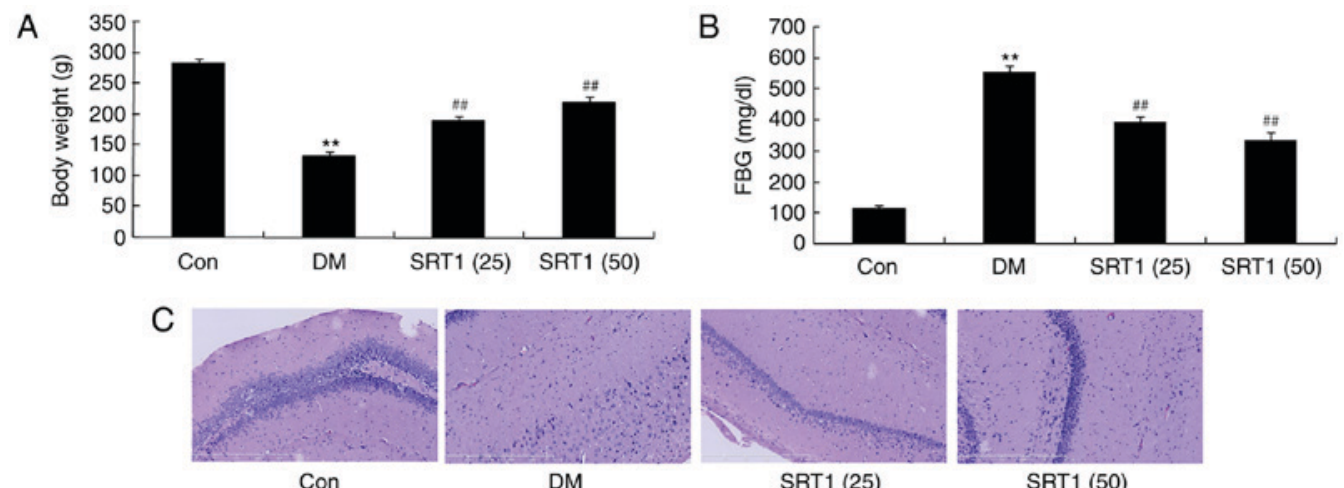

Figure 1. Effect of SRT1720 on body weight and FBG in a rat model of type 2 DM. (A) Body weight, (B) FBG levels, and (C) hippocampus analysis via H\&E staining in control, DM and SRT1720-treated DM rats. ${ }^{* *} \mathrm{P}<0.01$ vs. control group; ${ }^{\# /} \mathrm{P}<0.01$ vs. DM group. FBG, fasting blood glucose; DM, diabetes mellitus; Con, control; SRT1 (25), 25 mg/kg SRT1720; SRT1 (50), 50 mg/kg SRT1720.
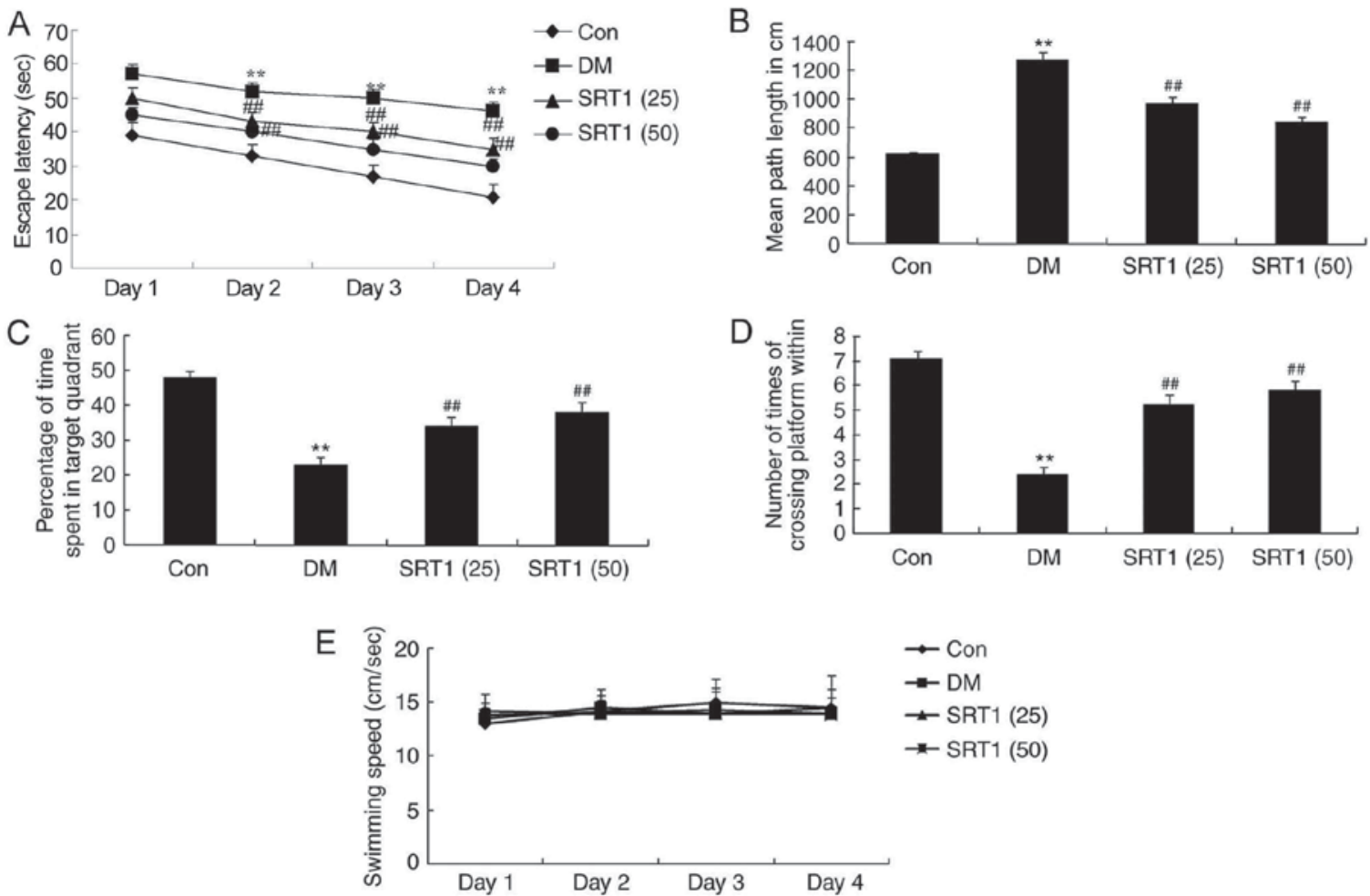

Figure 2. Effect of SRT1720 on cognitive function in a rat model of type 2 DM. (A) Escape latency, (B) mean path length, (C) percentage of time spent in the target quadrant, (D) the number of times animals crossed the former platform location for day 5 and (E) swimming speed were determined using Morris water maze testing on control, DM and SRT1720-treated DM rats. ${ }^{* *} \mathrm{P}<0.01$ vs. control group; ${ }^{\# \#} \mathrm{P}<0.01$ vs. DM group. DM, diabetes mellitus; Con, control; SRT1 (25), $25 \mathrm{mg} / \mathrm{kg}$ SRT1720; SRT1 (50), $50 \mathrm{mg} / \mathrm{kg}$ SRT1720.

SRT1720 improves cognitive function. In the Morris water maze testing, a significantly increased escape latency time was recorded in DM rats after 2-4 days of training, compared with control rats (Fig. 2A). Compared with the DM group, SRT1720 treatment significantly reduced the escape latency time (Fig. 2A). Additionally, the mean path length was notably increased in the DM group compared with the control group rats after 5 days of training; SRT1720 treatment reversed this effect and the mean path length was significantly reduced compared with the DM rats (Fig. 2B). Furthermore, on day 5 , DM rats spent significantly less time in the target quadrant compared with the control group rats (Fig. 2C). The frequency that the animals crossed the former platform location was also markedly reduced in DM rats compared with control rats (Fig. 2D). SRT1720 treatment in DM rats significantly reversed these alterations to results that were similar to those of the control group rats (Fig. 2C and D). No significant difference in swimming speed was determined among experimental groups (Fig. 2E). Taken together, these results demonstrated that SRT1720 may reduce cognitive impairment in rats with T2DM.

SRT1720 reverses DM-induced alterations in GSH-PX, GSH, $S O D$ and MDA levels. Compared with the control group, the results of ELISA demonstrated that the levels of GSH-PX (Fig. 3A), GSH (Fig. 3B) and SOD (Fig. 3C) were notably 
A
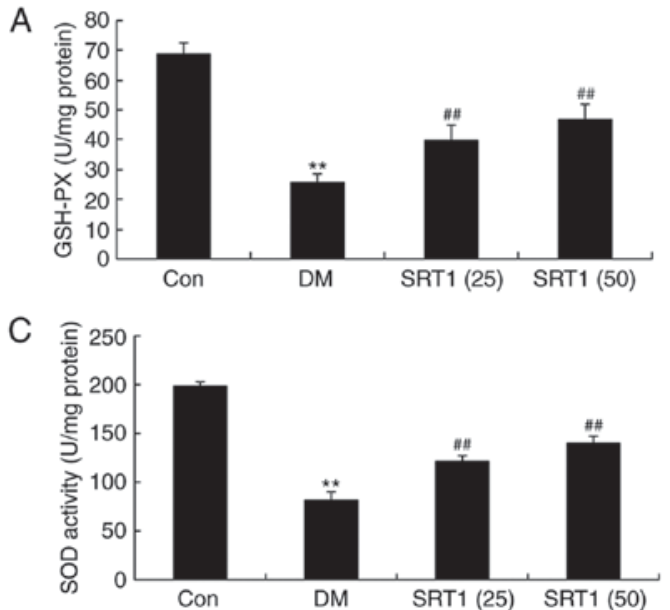

B

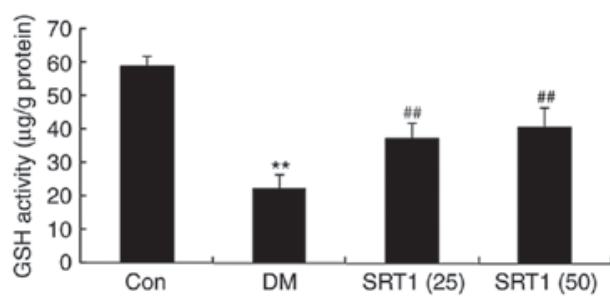

D

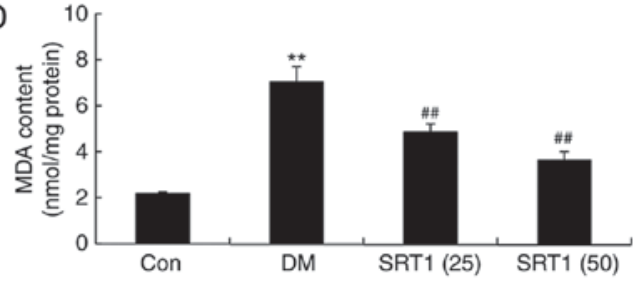

Figure 3. Effect of SRT1720 on DM-induced alterations in GSH-PX, GSH, SOD and MDA levels in hippocampal tissue of a rat model of type 2 DM. ELISA was performed to determine the levels of (A) GSH-PX, (B) GSH, (C) SOD and (D) MDA in control, DM and SRT1720-treated DM rats. ${ }^{* *}$ P $<0.01$ vs. control group; ${ }^{\# \#} \mathrm{P}<0.01$ vs. DM group. DM, diabetes mellitus; GSH, glutathione; GSH-PX, glutathione peroxidase; SOD, superoxide dismutase; MDA, malondialdehyde; Con, control; SRT1 (25), 25 mg/kg SRT1720; SRT1 (50), 50 mg/kg SRT1720.

A

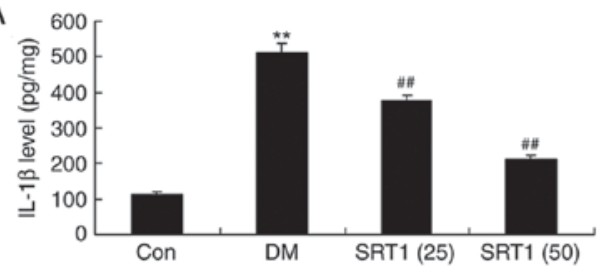

$\mathrm{C}$

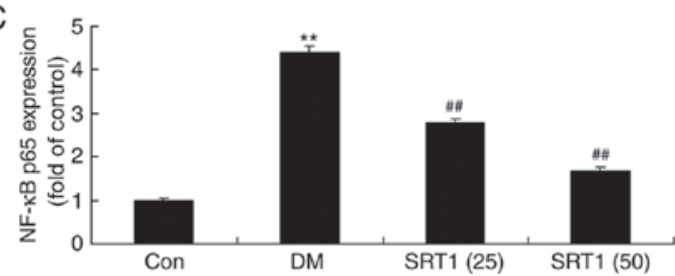

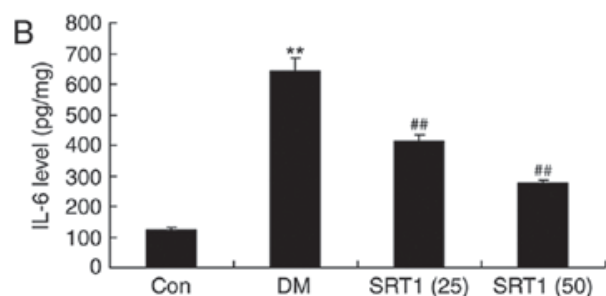

D

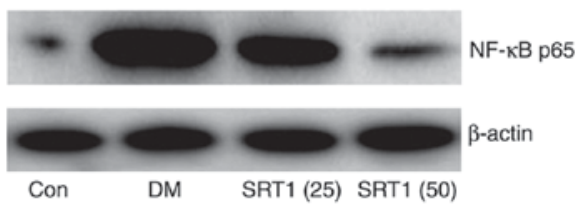

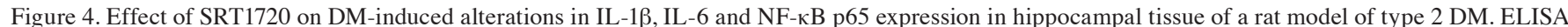
was performed to determine the levels of (A) IL-1 $\beta$ and (B) IL-6 in control, DM and SRT1720-treated DM rats. (C) Densitometric analysis was performed on western blotting results to quantify NF- $\mathrm{kB}$ p65 expression in control, DM and SRT1720-treated DM rats. (D) Representative western blot bands for NF-kB p65 protein expression in each group. ${ }^{* *} \mathrm{P}<0.01$ vs. control group; ${ }^{\#} \mathrm{P}<0.01$ vs. DM group. DM, diabetes mellitus; IL, interleukin; NF- $\mathrm{kB}$, nuclear factor- $\mathrm{kB}$; Con, control; SRT1 (25), 25 mg/kg SRT1720; SRT1 (50), 50 mg/kg SRT1720.

reduced in DM rats. However, SRT1720 treatment significantly increased these levels compared with the DM group (Fig. 3A-C). By contrast, MDA levels were significantly increased in DM rats compared with the control group, and SRT1720 treatment markedly reduced this MDA content in DM rats (Fig. 3D). These results indicate that SRT1720 may reduce the level of oxidative stress in rats with T2DM.

SRT1720 reduces $N F-\kappa B$ p $65, I L-1 \beta$ and IL-6 expression in DM rats. Compared with the control group, ELISA results demonstrated that IL-1 $\beta$ (Fig. 4A) and IL-6 (Fig. 4B) expression was significantly increased in DM rats. In addition, western blot analysis demonstrated that the protein expression of NF- $\mathrm{kB}$ p65 in DM rats was also significantly increased compared with the control group (Fig. 4C and D). SRT1720 treatment significantly reversed these effects and the expression of these proteins was significantly reduced compared with DM rats. These results indicate that SRT1720 may reduce inflammation in T2DM rats.
SRT1720 increases eNOS, PPAR $\gamma$ and AMPK expression. The protein expression of eNOS, PPAR $\gamma$ and AMPK was detected by western blot analysis (Fig. 5A). Compared with the control group, eNOS (Fig. 5A and B), PPAR $\gamma$ (Fig. 5A and C) and AMPK (Fig. 5A and D) protein expression was significantly reduced in the DM model rats. However, SRT1720 treatment significantly increased eNOS, PPAR $\gamma$ and AMPK protein expression in DM rats.

SRT1720 decreases the activity of caspase-3. Caspase-3 activity was significantly upregulated in DM rats compared with the control group. Additionally, compared with the DM group, caspase-3 activity was markedly downregulated in the SRT1720 treatment groups (Fig. 6).

Effect of SRT1720 on the protein expression of HSP70, SIRT1, Nrf2 and HO-1. The protein expression of HSP70, SIRT1, Nrf2 and HO-1 was determined by western blot 

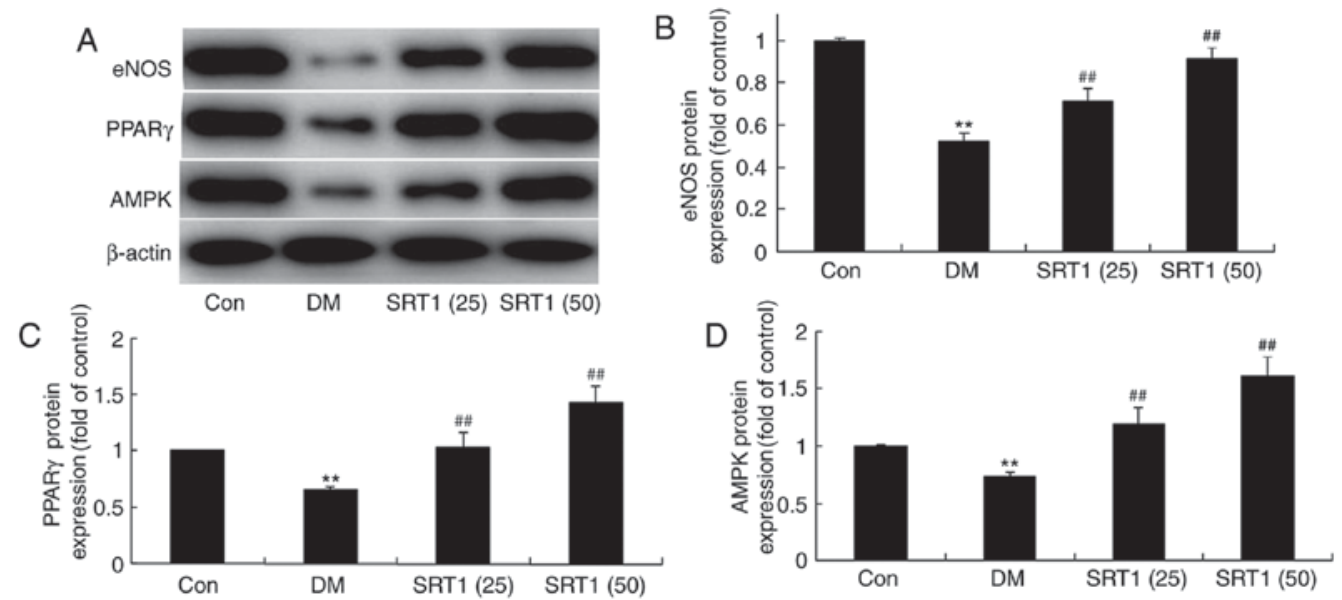

Figure 5. Effect of SRT1720 on DM-induced alterations in hippocampal tissue of DM rats. (A) Protein expression levels of eNOS, PPAR $\gamma$ and AMPK were detected by western blot analysis. Densitometric analysis of western blotting results was performed to quantify the protein levels of (B) eNOS, (C) PPAR $\gamma$ and (D) AMPK in control, DM and SRT1720-treated DM rats. The results demonstrated that the expression of eNOS, PPAR $\gamma$ and AMPK was increased in the SRT1720-treated groups compared with the DM group. ${ }^{* *} \mathrm{P}<0.01$ vs. control group; ${ }^{\# \#} \mathrm{P}<0.01$ vs. DM group. DM, diabetes mellitus; eNOS, endothelial nitric oxide synthase; PPAR $\gamma$, peroxisome proliferator-activated receptor $\gamma$; AMPK, AMP-activated protein kinase; Con, control; SRT1 (25), 25 mg/kg SRT1720; SRT1 (50), $50 \mathrm{mg} / \mathrm{kg}$ SRT1720.

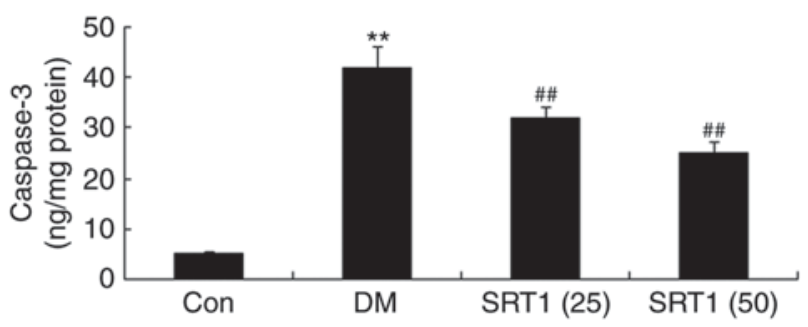

Figure 6. Effect of SRT1720 on caspase-3 activity in hippocampal tissue of DM rats. A caspase-3 activity assay kit was used to determine the activity of caspase-3 in control, DM and SRT1720-treated DM rats. ${ }^{* *} \mathrm{P}<0.01$ vs. control group; ${ }^{\#} \mathrm{P}<0.01$ vs. DM group. DM, diabetes mellitus; Con, control; SRT1 (25), 25 mg/kg SRT1720; SRT1 (50), 50 mg/kg SRT1720.

analysis (Fig. 7A). It was revealed that HSP70 expression was significantly increased (Fig. 7B), while SIRT1 (Fig. 7C), Nrf2 (Fig. 7D) and HO-1 (Fig. 7E) expression was markedly suppressed, in the DM group compared with the control group. Furthermore, compared with the DM group, HSP70 expression was significantly reduced (Fig. 7B), and SIRT1, Nrf2 and HO-1 expression was markedly increased, in the SRT1720 treatment groups. These results indicate that SRT1720 may reduce cognitive decline in diabetic rats through antioxidative and anti-inflammatory mechanisms, potentially via a SIRT1/Nrf2-NF- $\mathrm{B}$ signaling pathway.

$N F-\kappa B$ inhibitor enhances the anti-inflammatory effects of

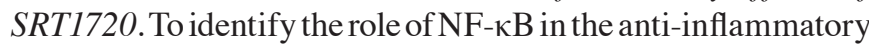
effects of SRT1720, the protein expression of NF- $\kappa B$, IL-1 $\beta$ and IL-6 was analyzed in a PC12 diabetic cell model treated with an NF- $\kappa$ B inhibitor (Fig. 8). ELISA was performed to measure IL-1 $\beta$ (Fig. 8A) and IL-6 (Fig. 8B) levels, and western blotting was performed to measure NF- $\kappa \mathrm{B}$ p65 levels (Fig. 8C and D). The results demonstrated that SRT1720 treatment significantly reduced NF- $\kappa$ B p 65, IL-1 $\beta$ and IL-6 expression compared with control diabetic cells. Furthermore, compared with SRT1720 treatment alone, combination treatment with an $\mathrm{NF}-\kappa \mathrm{B}$ inhibitor further decreased the expression of these proteins in PC12 diabetic model cells.

Nrf2 enhances the antioxidative effects of SRT1720. To identify the role of Nrf2 in the antioxidative effects of SRT1720, the protein expression of HO-1 and Nrf2 was detected in a PC12 diabetic cell model treated with Nrf2 agonist using western blot analysis (Fig. 9). Compared with the control diabetic cells, SRT1720 treatment significantly increased the protein expression of HO-1 and Nrf2, while combined treatment with SRT1720 and Nrf2 agonist further increased the expression of Nrf2 and HO-1 (Fig. 9). Additionally, the levels of GSH-PX (Fig. 10A), GSH (Fig. 10B), SOD (Fig. 10C) and MDA (Fig. 10D) were detected by ELISA in a PC12 diabetic cell model treated with $\mathrm{Nrf} 2$ agonist. Compared with the control group, GSH-PX, GSH and SOD levels were significantly increased in the SRT1720 treatment group (Fig. 10A-C), an effect that was enhanced in the combination treatment group. By contrast, MDA levels were significantly decreased in PC12 cells treated with SRT1720, compared with control group, and combination treatment with Nrf2 agonist further decreased MDA levels (Fig. 10D).

\section{Discussion}

Population ageing and geriatric disease have become important social and medical problems as social economy and medical science have advanced (10). Among the various types of geriatric disease, the incidence of cognitive impairment has distinctly increased, leading to severe impairments in the quality of life of patients and heavy burdens to the families of patients and society (11). The present study demonstrated that SRT1720 reversed reductions in body weight, reduced FBG and improved cognitive function in a rat model of T2DM. Furthermore, SRT1720 upregulated GSH-PX, GSH and SOD levels, and downregulated levels of MDA, in DM rats. Consistent with the findings of the present study, Ding et al (12) reported that improvements observed in rat cognitive deficits 


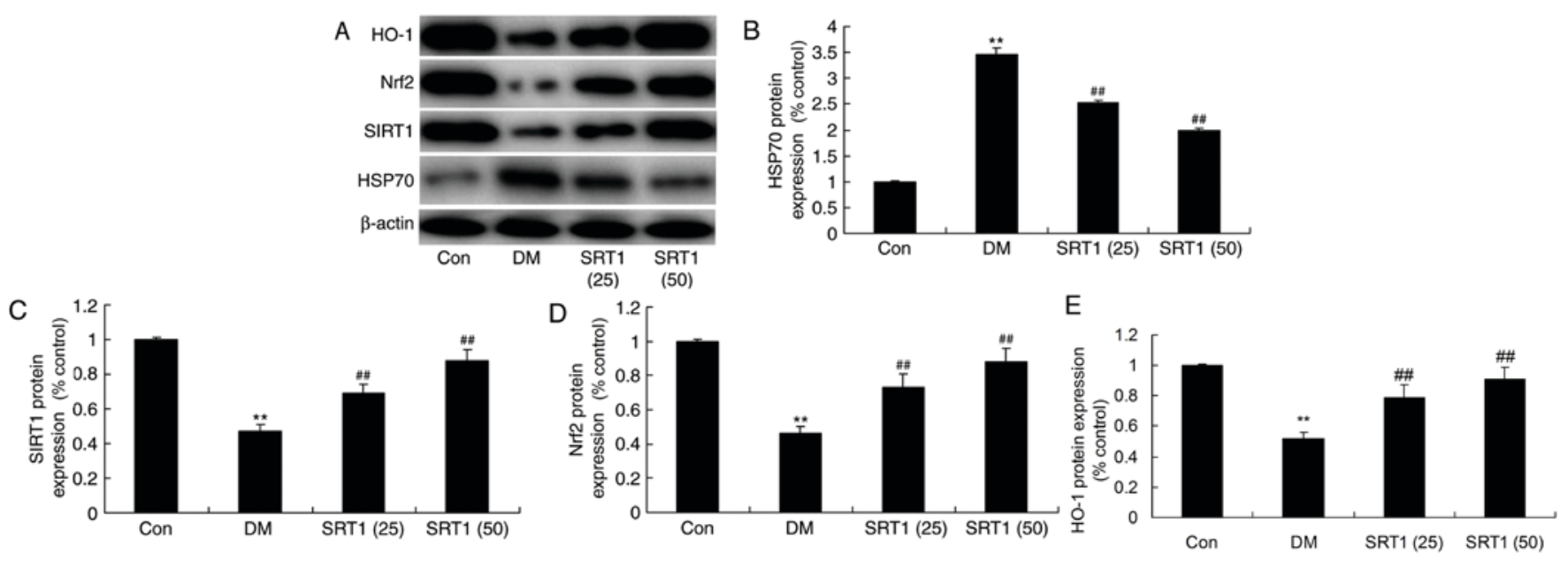

Figure 7. Effect of SRT1720 on DM-induced alterations in HSP70, SIRT1, Nrf2 and HO-1 protein expression in hippocampal tissue. (A) Protein expression levels of HSP70, SIRT1, Nrf2 and HO-1 were detected by western blot analysis. Densitometric analysis of western blotting results was performed to quantify the protein levels of (B) HSP70, (C) SIRT1, (D) Nrf2 and (E) HO-1 in control, DM and SRT1720-treated DM rats. The results demonstrated that alterations observed in DM rats compared with control rats were reversed by SRT1720 treatment. ${ }^{* *} \mathrm{P}<0.01$ vs. control group; ${ }^{\# \#} \mathrm{P}<0.01$ vs. DM group. DM, diabetes mellitus; HSP70, heat shock 70 kDa protein; SIRT1, sirtuin 1; Nrf2, nuclear factor erythroid 2-related factor 2; HO-1, heme oxygenase 1; Con, control; SRT1 (25), $25 \mathrm{mg} / \mathrm{kg}$ SRT1720; SRT1 (50), $50 \mathrm{mg} / \mathrm{kg}$ SRT1720.
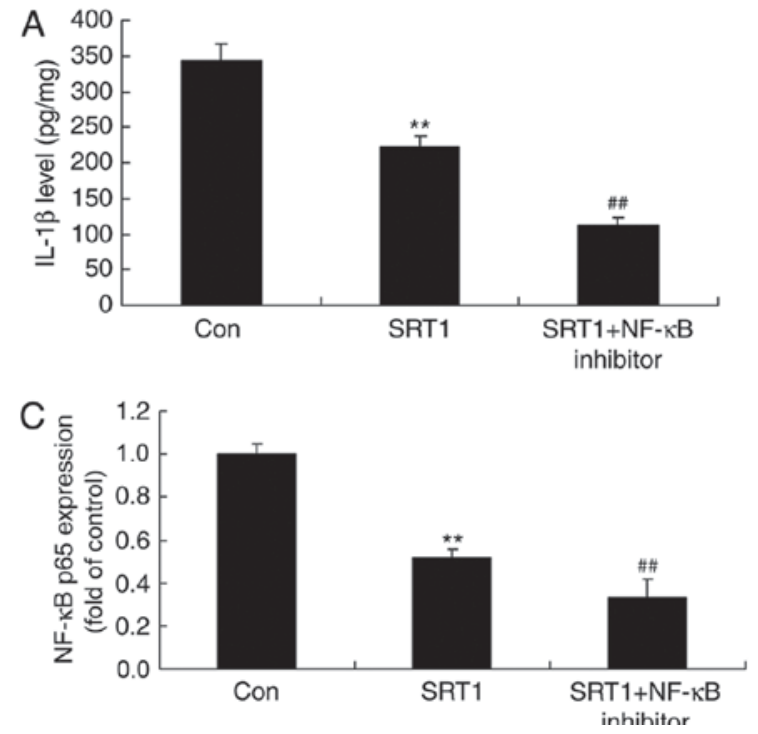

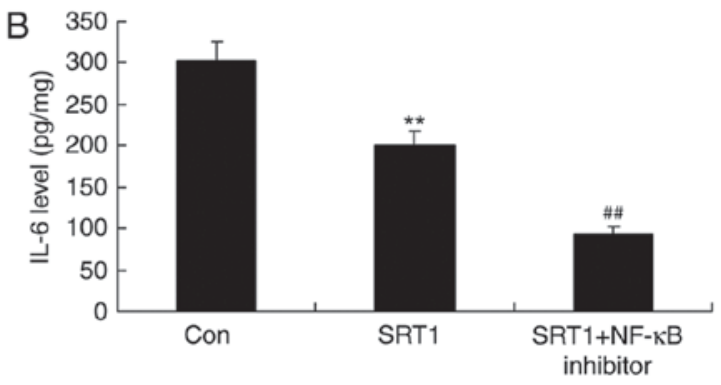

D

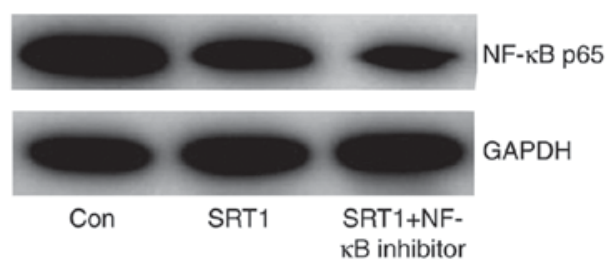

Figure 8. Effect of an NF-кB inhibitor on the anti-inflammatory action of SRT1720 in PC12 diabetic model cells. ELISA was performed to determine the levels of (A) IL-1 $\beta$ and (B) IL-6 in control, SRT1720-treated and SRT1720 + NF- $\mathrm{BB}$ inhibitor-treated PC12 diabetic model cells. (C) Densitometric analysis was

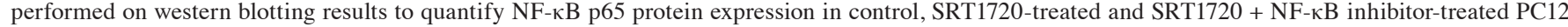
diabetic model cells. (D) Representative western blot bands for NF- $\mathrm{KB}$ p65 protein expression in each group. JSH-23 was employed as the NF- $\mathrm{kB}$ inhibitor.

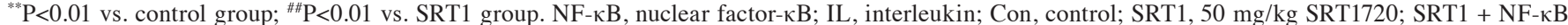
inhibitor, $50 \mathrm{mg} / \mathrm{kg} \mathrm{SRT} 1720+2 \mu \mathrm{M} \mathrm{JSH}-23$.

following hyperbaric oxygen preconditioning was mediated by SIRT1. These results demonstrate that SRT1720 may have potential as a novel drug for cognitive impairment in diabetes.

$\mathrm{NF}-\kappa \mathrm{B}$ is a major immunomodulatory factor that has important roles in cells and peripheral body fluids, and is among the strongest immunomodulatory factors within the body (13). T2DM pathogenesis typically involves b cell injury in the islet of Langerhans, which is caused by the chronic activation of nonspecific immunity by increased blood glucose, saturated fatty acid and adipose tissue levels (14). In the present study, the rat tissues were analyzed by western blotting. The results of the present study revealed that SRT1720 significantly downregulated NF- $\mathrm{B}$ and upregulated eNOS expression in DM rats.

AMPK is involved in the regulation of glycometabolism and fat metabolism, and leads to effects on various functions, including energy metabolism and signal transduction (15). AMPK activity is regulated and controlled by the AMP/ATP ratio (16). The present study revealed that SRT1720 markedly increased PPAR $\gamma$ and AMPK protein expression, and reduced caspase-3 activity, in DM rats. Yang et al (15) demonstrated that the upregulation of SIRT1-AMPK ameliorated liver injury in hepatic stellate cells through PPAR $\gamma$ expression. AMPK activity is a major regulator of metabolic homeostasis, which 

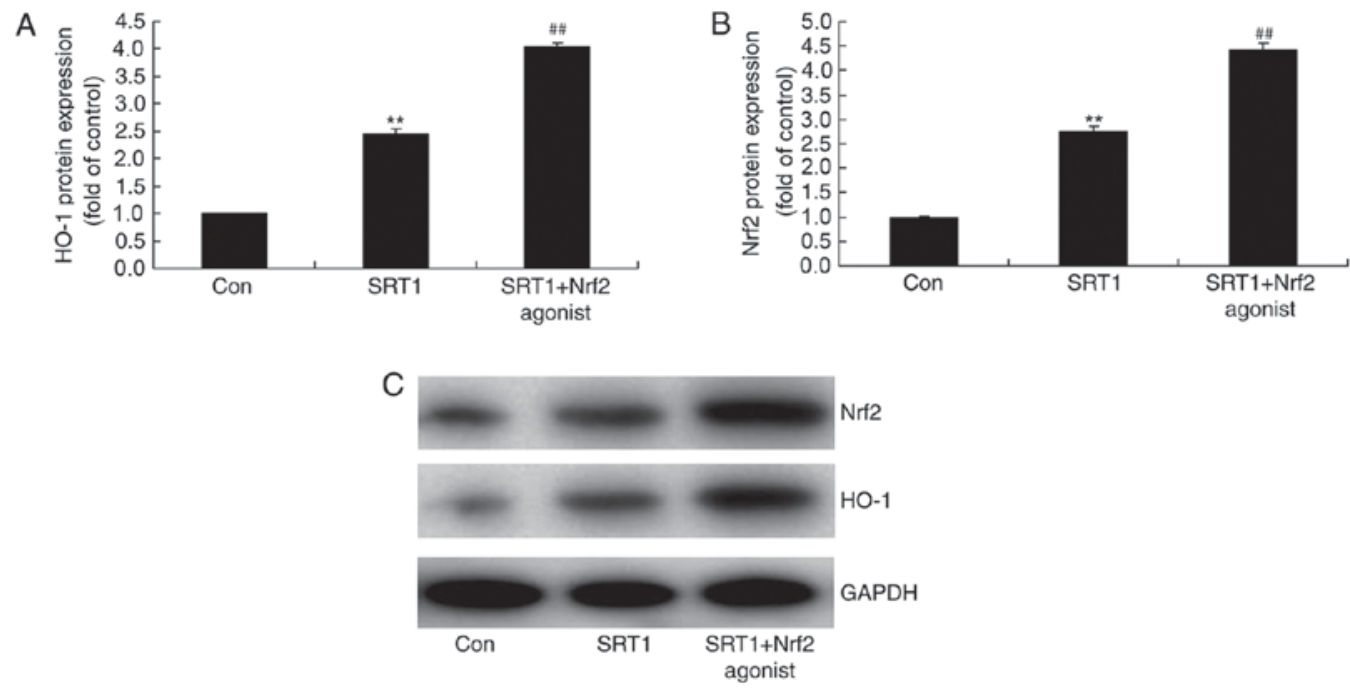

Figure 9. Effect of a Nrf2 agonist on the antioxidative action of SRT1720 in PC12 diabetic model cells. Densitometric analysis was performed on western blotting results to quantify (A) HO-1 and (B) Nrf2 protein expression in control, SRT1720-treated and SRT1720 + Nrf2 agonist-treated PC12 diabetic model cells. (C) Representative western blot bands for HO-1 and Nrf2 protein expression in each group. Curcumin was employed as the Nrf2 agonist. ${ }^{* *} \mathrm{P}<0.01$ vs. control group; ${ }^{\# /} \mathrm{P}<0.01$ vs. SRT1 group. Nrf2, nuclear factor erythroid 2-related factor 2; HO-1, heme oxygenase 1; Con, control; SRT1, $50 \mathrm{mg} / \mathrm{kg}$ SRT1720; SRT1 + Nrf2 agonist, $50 \mathrm{mg} / \mathrm{kg}$ SRT1720 $+25 \mu \mathrm{M}$ curcumin.
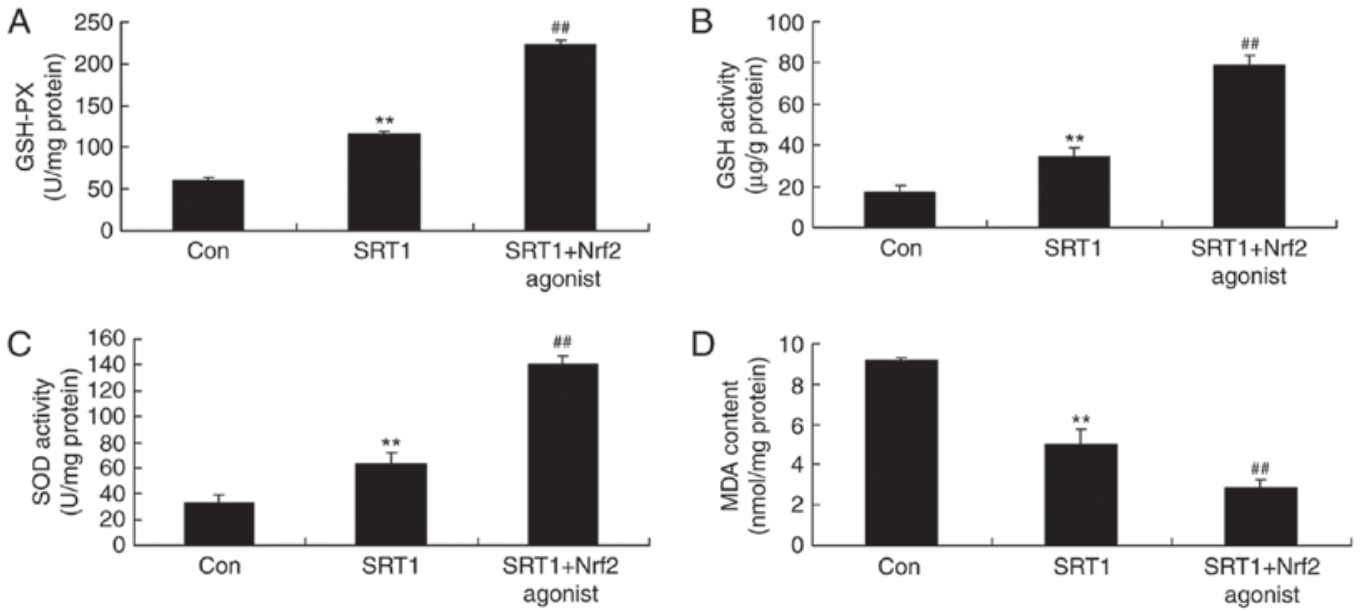

Figure 10. Effect of a Nrf2 agonist on GSH-PX, GSH, SOD and MDA levels in PC12 diabetic model cells treated with SRT1720. ELISA was performed to determine the levels of (A) GSH-PX, (B) GSH, (C) SOD and (D) MDA in control, SRT1720-treated and SRT1720 + Nrf2 agonist-treated PC12 diabetic model cells. Curcumin was employed as the Nrf2 agonist. ${ }^{* *} \mathrm{P}<0.01$ vs. control group; ${ }^{* \#} \mathrm{P}<0.01$ vs. SRT1 group. Nrf2, nuclear factor erythroid 2-related factor 2; GSH, glutathione; GSH-PX, glutathione peroxidase; SOD, superoxide dismutase; MDA, malondialdehyde; Con, control; SRT1, 50 mg/kg SRT1720; SRT1 + Nrf2 agonist, $50 \mathrm{mg} / \mathrm{kg}$ SRT1720 $+25 \mu \mathrm{M}$ curcumin.

is regulated by reactive oxygen species (17). The present study revealed that SRT1720 increased eNOS and AMPK expression in DM rats. Similar results were reported by Liu et al (18), who demonstrated that HSP70 protected mice against lung ischemia/reperfusion injury through the SIRT1/AMPK/eNOS signaling pathway.

The antioxidative and anti-neurotoxic effects of Nrf2 have been widely recognized $(5,19)$. Additionally, therapy targeting the kelch-like ECH-associated protein 1 (Keap1)-Nrf2-antioxidant response element (ARE) signaling pathway has become the focus of research at present (19). It has been suggested that inhibiting the Keap1-Nrf2-ARE pathway may result in endothelial dysfunction, vascular endothelial dysfunction and insulin resistance (19). Therefore, regulation of $\mathrm{Nrf} 2$ expression is expected to be a potential means for the prevention and treatment of diabetes and its complications. The present study demonstrated that SRT1720 treatment significantly reversed the inhibition of $\mathrm{Nrf} 2$ and HO-1 expression observed in DM rats. Furthermore, when SRT1720 was combined with a Nrf2 agonist, curcumin, Nrf2 and $\mathrm{HO}-1$ expression was further induced in PC12 cells treated with SRT1720. Additionally, the Nrf2 agonist increased the levels of GSH-PX, GSH and SOD, and inhibited MDA levels, in PC12 cells treated with SRT1720. Xue et al (20) reported that SIRT1 may be involved in a Nrf2/antioxidant defense pathway against transient focal cerebral ischemia. SRT1720 may also regulate the Nrf2/HO-1/antioxidant pathway in diabetic cognitive impairment. Liu et al (21) demonstrated that licochalcone A reduced oxygen-glucose deprivation/reperfusion damage by attenuating oxidative 
stress injury and the inflammatory response via SIRT1/Nrf2 signaling in rat primary cortical neurons. The results of the present study revealed that SRT1720 may regulate the SIRT1/Nrf2 pathway to inhibit oxidative stress and cognitive dysfunction in diabetes.

SIRT1 is essential for normal cognitive function and synaptic plasticity (22). It has been demonstrated that SIRT1-knockout mice exhibit immediate memory defects, short-term and long-term associative memory impairment, dendritic tree branching of hippocampal neuron and reductions in neurite length and complexity. This suggests that SIRT1 may be essential in normal spatial learning and the regulation of synaptic plasticity (23). Long-term calorie restriction has been reported to inhibit NF- $\kappa \mathrm{B}$, thus reducing its proinflammatory effect (24). The present study revealed that an $\mathrm{NF}-\kappa \mathrm{B}$ inhibitor, JSH-23, further suppressed NF- $\mathrm{NB}$ expression, as well as IL-1 $\beta$ and IL-6 levels, in PC12 cells treated with SRT1720. Liu et al (25) reported that SIRT1 may mediate $\mathrm{NF}-\kappa \mathrm{B}$ in human alveolar epithelial cells. Taken together, these results demonstrate that SRT1720 may regulate the inflammatory defense system to suppress $\mathrm{NF}-\mathrm{\kappa B}$ and subsequently reduce cognitive impairment in diabetes.

In conclusion, the results of the present study indicate that SRT1720 may possess antioxidant and anti-inflammatory properties and may reduce cognitive decline through a Nrf2-NF- $\kappa$ B-dependent mechanism in T2DM rats. These findings indicate that SRT1720 may have potential as a drug for the treatment of cognitive decline in diabetes.

\section{Acknowledgements}

Not applicable.

\section{Funding}

No funding was received.

\section{Availability of data and materials}

The analyzed data sets generated during the study are available from the corresponding author on reasonable request.

\section{Authors' contributions}

QZ made substantial contributions to the design of the study; FW, YS, RZ and XG performed the experiments; QZ and FW analyzed the data; QZ wrote the manuscript.

\section{Ethics approval and consent to participate}

The present study was approved by the Ethics Committee of Chinese PLA General Hospital.

\section{Patient consent for publication}

Not applicable.

\section{Competing interests}

The authors declare that they have no competing interests.

\section{References}

1. Shikora S, Toouli J, Herrera MF, Kulseng B, Zulewski H, Brancatisano R, Kow L, Pantoja JP, Johnsen G, Brancatisano A, et al: Vagal blocking improves glycemic control and elevated blood pressure in obese subjects with type 2 diabetes mellitus. J Obes 2013: 245683, 2013.

2. Fonseca VA, Ferrannini E, Wilding JP, Wilpshaar W, Dhanjal P, Ball G and Klasen S: Active- and placebo-controlled dose-finding study to assess the efficacy, safety and tolerability of multiple doses of ipragliflozin in patients with type 2 diabetes mellitus. J Diabetes Complications 27: 268-273, 2013.

3. Liang L, Stone RC, Stojadinovic O, Ramirez H, Pastar I, Maione AG, Smith A, Yanez V, Veves A, Kirsner RS, et al: Integrative analysis of miRNA and mRNA paired expression profiling of primary fibroblast derived from diabetic foot ulcers reveals multiple impaired cellular functions. Wound Repair Regen 24: 943-953, 2016.

4. Zhang J, Sun XJ, Chen J, Hu ZW, Wang L, Gu DM and Wang AP: Increasing the miR-126 expression in the peripheral blood of patients with diabetic foot ulcers treated with maggot debridement therapy. J Diabetes Complications 31: 241-244, 2017.

5. Madhyastha R, Madhyastha H, Pengjam Y, Nakajima Y Omura $\mathrm{S}$ and Maruyama M: NFkappaB activation is essential for miR-21 induction by TGF $\beta 1$ in high glucose conditions. Biochem Biophys Res Commun 451: 615-621, 2014.

6. Wang T, He R, Zhao J, Mei JC, Shao MZ, Pan Y, Zhang J, Wu HS, Yu M, Yan WC, et al: Negative pressure wound therapy inhibits inflammation and upregulates activating transcription factor-3 and downregulates nuclear factor-kB in diabetic patients with foot ulcerations. Diabetes Metab Res Rev 33, 2017. doi: $10.1002 / \mathrm{dmrr} .2871$.

7. Bhat MA and Gandhi G: Elevated oxidative DNA damage in patients with coronary artery disease and its association with oxidative stress biomarkers. Acta Cardiol: 1-8, 2018 (Epub ahead of print).

8. Yang B, Xu B, Zhao H, Wang YB, Zhang J, Li CW, Wu Q, Cao YK, Li Y and Cao F: Dioscin protects against coronary heart disease by reducing oxidative stress and inflammation via Sirt1/Nrf2 and p38 MAPK pathways. Mol Med Rep 18: 973-980, 2018.

9. Milne JC, Lambert PD, Schenk S, Carney DP, Smith JJ, Gagne DJ, Jin L, Boss O, Perni RB, Vu CB, et al: Small molecule activators of SIRT1 as therapeutics for the treatment of type 2 diabetes. Nature 450: 712-716, 2007.

10. Wilmot EG, Davies MJ, Edwardson CL, Gorely T, Khunti K, Nimmo M, Yates T and Biddle SJ: Rationale and study design for a randomised controlled trial to reduce sedentary time in adults at risk of type 2 diabetes mellitus: Project stand (Sedentary Time ANd diabetes). BMC Public Health 11: 908, 2011.

11. Shah PS, Todkar JS and Shah SS: Effectiveness of laparoscopic sleeve gastrectomy on glycemic control in obese Indians with type 2 diabetes mellitus. Surg Obes Relat Dis 6: 138-141, 2010.

12. Ding P, Ren D, He S, He M, Zhang G, Chen Y, Sang H, Peng Z and Yan W: Sirt1 mediates improvement in cognitive defects induced by focal cerebral ischemia following hyperbaric oxygen preconditioning in rats. Physiol Res 66: 1029-1039, 2017.

13. Palmer R, Nyman E, Penney M, Marley A, Cedersund G and Agoram B: Effects of IL-1 $\beta$-blocking therapies in type 2 diabetes mellitus: A quantitative systems pharmacology modeling approach to explore underlying mechanisms. CPT Pharmacometrics Syst Pharmacol 3: e118, 2014.

14. Raja L, Palanivelu S and Panchanatham S: Anti-inflammatory property of Kalpaamruthaa on myocardium in type 2 diabetes mellitus induced cardiovascular complication. Immunopharmacol Immunotoxicol 35: 119-125, 2013.

15. Yang J, Zhao P, Wan D, Zhou Q, Wang C, Shu G, Mei Z and Yang X: Antidiabetic effect of methanolic extract from berberis julianae Schneid. via activation of AMP-activated protein kinase in type 2 diabetic mice. Evid Based Complement Alternat Med 2014: 106206, 2014

16. Russo GL, Russo M and Ungaro P: AMP-activated protein kinase: A target for old drugs against diabetes and cancer. Biochem Pharmacol 86: 339-350, 2013.

17. Yang RH, Lin J, Hou XH, Cao R, Yu F, Liu HQ, Ji AL, Xu XN, Zhang L and Wang F: Effect of docosahexaenoic acid on hippocampal neurons in high-glucose condition: Involvement of $\mathrm{PI} 3 \mathrm{~K} / \mathrm{AKT} /$ nuclear factor-kB-mediated inflammatory pathways. Neuroscience 274: 218-228, 2014. 
18. Liu S, Xu J, Fang C, Shi C, Zhang X, Yu B and Yin Y: Over-expression of heat shock protein 70 protects mice against lung ischemia/reperfusion injury through SIRT1/AMPK/eNOS pathway. Am J Transl Res 8: 4394-4404, 2016.

19. Kuo YR, Chien CM, Kuo MJ, Wang FS, Huang EY and Wang CJ: Endothelin-1 expression associated with lipid peroxidation and nuclear factor- $\mathrm{kB}$ activation in type 2 diabetes mellitus patients with angiopathy and limb amputation. Plast Reconstr Surg 137: $187 \mathrm{e}-195 \mathrm{e}, 2016$

20. Xue F, Huang JW, Ding PY, Zang HG, Kou ZJ, Li T, Fan J, Peng ZW and Yan WJ: Nrf2/antioxidant defense pathway is involved in the neuroprotective effects of Sirtl against focal cerebral ischemia in rats after hyperbaric oxygen preconditioning. Behav Brain Res 309: 1-8, 2016.

21. Liu X, Ma Y, Wei X and Fan T: Neuroprotective effect of licochalcone A against oxygen-glucose deprivation/reperfusion in rat primary cortical neurons by attenuating oxidative stress injury and inflammatory response via the SIRT1/Nrf2 pathway. J Cell Biochem 119: 3210-3219, 2018.
22. Tsarouhas K, Tsitsimpikou C, Papantoni X, Lazaridou D, Koutouzis M, Mazzaris S, Rezaee R, Mamoulakis C, Georgoulias P, Nepka C, et al: Oxidative stress and kidney injury in trans-radial catheterization. Biomed Rep 8: 417-425, 2018.

23. Prasad K and Dhar I: Oxidative stress as a mechanism of added sugar-induced cardiovascular disease. Int J Angiol 23: 217-226, 2014.

24. Bicer M, Senturk T, Yanar M, Tutuncu A, Oral AY, Ulukaya E, Serdar Z and Signak IS: Effects of off-pump versus on-pump coronary artery bypass grafting: Apoptosis, inflammation and oxidative stress. Heart Surg Forum 17: E271-E276, 2014.

25. Liu X, Yang T, Sun T and Shao K: SIRT1-mediated regulation of oxidative stress induced by Pseudomonas aeruginosa lipopolysaccharidesinhumanalveolarepithelialcells.MolMedRep 15:813-818, 2017.

This work is licensed under a Creative Commons Attribution-NonCommercial-NoDerivatives 4.0 International (CC BY-NC-ND 4.0) License. 